\title{
Gravitational acceleration as a cue for absolute size and distance?
}

\author{
HEIKO HECHT \\ Universität Bielefeld, Bielefeld, Germany \\ MARY K. KAISER \\ NASA Ames Research Center, Moffett Field, Califormia \\ and \\ MARTIN S. BANKS \\ University of Califormia, Berkeley, California
}

\begin{abstract}
When an object's motion is influenced by gravity, as in the rise and fall of a thrown ball, the vertical component of acceleration is roughly constant at $9.8 \mathrm{~m} / \mathrm{sec}^{2}$. In principle, an observer could use this information to estimate the absolute size and distance of the object (Saxberg, 1987a; Watson, Banks, von Hofsten, \& Royden, 1992). In five experiments, we examined people's ability to utilize the size and distance information provided by gravitational acceleration. Observers viewed computer simulations of an object rising and falling on a trajectory aligned with the gravitational vector. The simulated objects were balls of different diameters presented across a wide range of simulated distances. Observers were asked to identify the ball that was presented and to estimate its distance. The results showed that observers were much more sensitive to average velocity than to the gravitational acceleration pattern. Likewise, verticality of the motion and visibility of the trajectory's apex had negligible effects on the accuracy of size and distance judgments.
\end{abstract}

People need to process the absolute distance and size of objects in order to act in the environment. For example, in order to catch and grasp a thrown ball successfully, a person must place his/her hands in the appropriate spatial position and in an appropriate conformation. To do so, he/she must use an appropriate combination of absolute variables (size of the ball, its distance, velocity, and temporal range). In the present paper, we address the question of how perceivers might pick up absolute distance and size information in such contexts.

The cues that an observer might employ to estimate absolute distance (and from that, to estimate absolute size) can be divided into those that are strictly monocular and those that are binocular as well. In principle, at least three monocular cues can be used to estimate absolute distance: accommodation, convergence, and familiar

The authors thank Geoffrey P. Bingham, Myron L. Braunstein, James A. Crowell, W. Farrell Edwards, and Walt Johnson for helpful discussion. John C. Baird, Hal A. Sedgwick, and one anonymous reviewer provided valuable comments on an earlier version of this paper. The research was supported by NASA RTOP 538-04-11 and by NSF Research Grant DBS-9309820 and AFOSR Grant F49620 to M.S.B. Portions of this research were presented in St. Louis at the annual meeting of the Psychonomic Society (November 1994). H. H. is with the Zentrum für interdisziplinaire Forschung at Bielefeld; M.S.B. is affiliated with both the School of Optometry and the Department of Psychology at Berkeley. Correspondence may be addressed to M. K. Kaiser, Mail Stop 262-2, NASA Ames Research Center, Moffett Field, CA 94035 (e-mail: moose@eos. arc.nasa.gov). size. However, none of these is very useful in general viewing situations: Human observers are not very sensitive to accommodation (Fisher \& Ciuffreda, 1988), convergence is only useful for objects at close range (von Hofsten, 1990), and familiar size requires knowledge of the object's actual size. Binocular information is also potentially useful. According to the conventional wisdom, stereopsis only specifies relative distance (i.e., depth); but it can actually specify absolute depth as well, if both horizontal and vertical disparities are employed efficiently (see, e.g., Gillam \& Lawergren, 1983; LonguetHiggins \& Mayhew, 1982). The evidence that human observers use such information accurately, however, is mixed (e.g., Cumming, Johnston, \& Parker, 1991; Rogers \& Bradshaw, 1993). Similarly, for certain object/observer motions, one can in principle estimate absolute distance from a combination of binocular disparity and motion parallax (e.g., Kellman \& Kaiser, 1995), but there is no empirical evidence that human observers can actually use this information.

Saxberg (1987a) and Watson, Banks, von Hofsten, and Royden (1992) suggested a novel monocular cue from which observers could estimate the absolute size and/or distance of objects whose motions are affected by gravity. Such cases include projectiles in flight, pendulum motion, fluid wave motion, and others. Saxberg, for example, showed that one can compute the distance, $Z$, to an object whose motion is governed by its initial velocity and gravity only. With perspective projection onto a plane parallel to the gravitational vector, 


$$
Z=g\left(2 v_{x}+a_{x} t\right) / 2\left(a_{x} v_{y}-a_{y} v_{x}\right),
$$

where $g$ is the gravitational constant, $v_{x}$ and $v_{y}$ are the retinal image velocities in the horizontal and vertical directions, respectively, and $a_{x}$ and $a_{y}$ are the retinal image accelerations in those same directions. Thus, in theory, one could estimate the absolute distance to an object from four retinal image variables: the vertical and horizontal components of the object's retinal velocity and the vertical and horizontal components of its retinal acceleration. Watson et al. (1992) and Tresilian (1994) have pointed out that the estimation of absolute distance is even simpler when the object's motion is vertical; the vertical retinal acceleration in that case is proportional to the gravitational constant, so Equation 1 becomes

$$
Z=g / a_{y} .
$$

Thus, one can in principle estimate the absolute distance to a freely falling object (without friction); the means of estimation is simpler if the observer knows that the object's motion is vertical.

The evidence concerning human observers' use of the gravity cue is mixed. Saxberg (1987b) reported that people cannot make reliable use of it, but Watson et al. (1992) pointed out that Saxberg's displays contained conflicting distance information. Thus, Saxberg's experiment did not provide persuasive evidence that human observers do not make good use of the gravity cue. There is both circumstantial and experimental evidence that people are indeed sensitive to the distance information in the dynamics of free fall. Cinematographers know that one can make miniature objects appear full sized by playing motion sequences at slower speed (e.g., Spottiswoode, 1969). Watson et al. (1992) pointed out that the rule of thumb employed in cinematography is exactly what one expects from the gravity-cue hypothesis. In a similar vein, Johansson and Jansson (1967) reported that observers can identify the motion sequence of divers jumping into a pool when the sequence is played at the normal speed rather than faster or slower than normal. However, the judgmental basis for these identifications is not clear: observers could have detected anomalies in the acceleration of the athlete's body as a whole or in any limb movements that were unnaturally fast or slow but unrelated to acceleration patterns. Finally, Muchisky and Bingham (1992) reported that observers could estimate the absolute size of free-falling objects reasonably accurately. Their stimuli were objects of constant projected (image) size; the objects' simulated distance (and therefore size) was varied by changing the dynamics of the falls. Although Muchisky and Bingham's observers may have used the distance information conveyed by the gravity cue, they might also have used other cues such as stimulus duration and final image velocity, which were confounded with simulated distance.

We conducted five experiments that were designed to test the gravity-cue hypothesis more directly. Observers were asked to estimate the absolute size and distance of simulated freely falling balls of various sizes and distances.
The results of Experiment 1 showed that observers' estimates were somewhat better than chance. Experiments 2 and 3 were designed to test the influence of potential artifacts; the results showed that observers' estimates in the task of Experiment 1 were, in part, based on the dynamics of free fall. Experiments 4 and 5 tested specific hypotheses and again found that observers used motion information, but not very accurately.

\section{EXPERIMENT 1}

In Experiment 1 $\mathrm{A}$, the animation sequence portrayed a ball launched upward from below the screen; thus, the ball was already in motion when it entered the field of view. There was no lateral component to the ball's motion. In Experiment 1B, only the downward part of the trajectory was shown. In other words, the animation sequence depicted a ball released from a point at the top of the display.

\section{Method}

Subjects. Eight male observers (ages 23-41) were paid for their participation. They had normal or corrected-to-normal vision. They were not informed of the experimental hypotheses.

Stimuli. The simulated objects were three balls: an official-sized basketball (diameter $=23.6 \mathrm{~cm})$, a child's basketball $(17.8 \mathrm{~cm})$, and an official-sized softball $(9.5 \mathrm{~cm})$. Each was presented at simulated distances of $2.5,5,7.5,10$, and $15 \mathrm{~m}$. Figure 1 depicts the projected sizes subtended on the display screen. The stimuli were generated on a Silicon Graphics Onyx $\mathrm{RE}^{2}$ workstation with a resolution of $1,280 \times 1,024$ pixels. The animation sequences were updated at the monitor's $60-\mathrm{Hz}$ refresh rate. The stimuli were presented by an Electrohome RGB rear-projection display system onto a $2.4 \times$

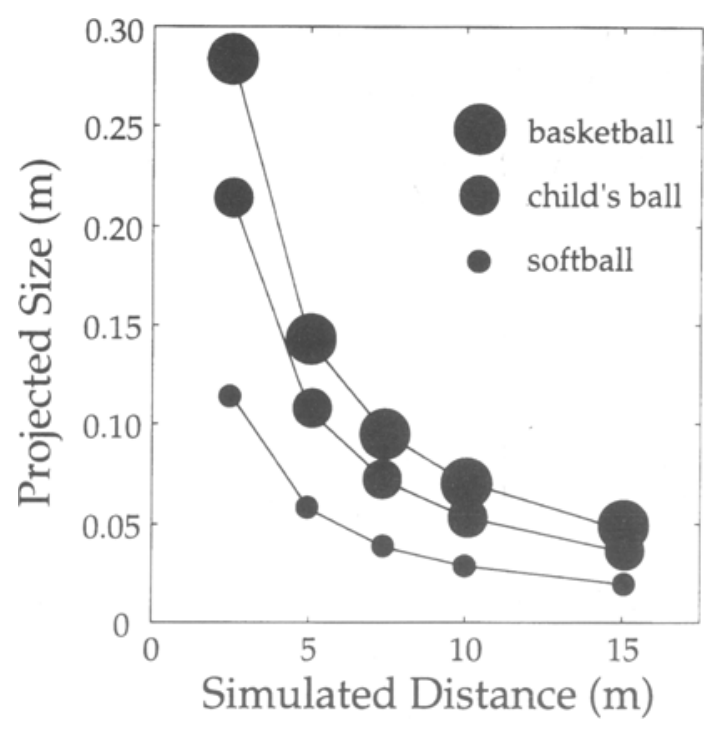

Figure 1. Projected sizes on the display screen for the three objects presented at the five simulated distances in Experiments $1-3$. The objects were balls with simulated diameters of $9.5,17.8$, and $23.6 \mathrm{~cm}$. The simulated distances were $2.5,5,7.5,10$, and $15 \mathrm{~m}$. Note that the ball went straight up and down without any lateral motion. 


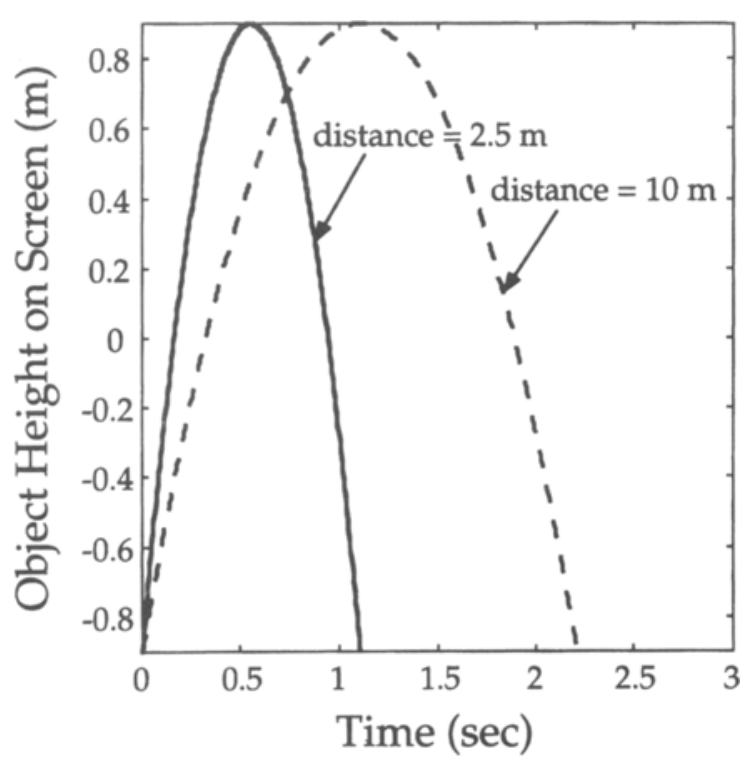

Figure 2. Projected trajectories in Experiment 1. Vertical position on the display screen is plotted as a function of time for objects presented at simulated distances of 2.5 and $10 \mathrm{~m}$. The objects were first visible at the bottom of the screen. They then rose to an apex at the top of the screen and dropped on the same path until they were no longer visible.

$1.8 \mathrm{~m}$ screen $\left(45^{\circ} \times 34^{\circ}\right)$. Figure 2 shows the screen height of the ball over time for two of the five simulated distances. The initial velocity was chosen so that the ball reached the apex of its trajectory near the top of the display. The total vertical displacement on the screen was, therefore, the same for all stimulus conditions. The viewing distance was $3 \mathrm{~m}$, and the displays were viewed monocularly. The room was completely dark except for the display. The edges of the screen were covered with black cloth. The simulated objects were dark blue, and the background was a dim gray. $\mathrm{Ob}$ servers initiated each animation sequence with a buttonpress.

Procedure. Without a good deal of practice, observers had difficulty in making both size and distance judgments after each stimulus presentation. Consequently, the sequence of stimuli for Experiment $1 \mathrm{~A}$ was presented twice, once with the observers making size judgments and once with the observers making distance judgments. For both sets of judgments, each possible combination of three ball sizes and five simulated distances was presented three times in random order.

Before viewing the animation sequences, the observers were given an actual softball, child's basketball, and official-sized basketball to handle. The experimenter showed the observers that all the balls fall at the same rate, and that the visual angle that the balls subtend depends on their distance. The observers were then seated $3 \mathrm{~m}$ from the projection screen in a dark room. The experimenter explained that simulations of the three balls in free flight at distances between 4 and $54 \mathrm{ft}$ would be shown. He also explained that the simulated balls would be textureless and identically colored.

In the first part of Experiments $1 \mathrm{~A}$ and $1 \mathrm{~B}$, the observer estimated the size of the simulated object; after each animation sequence was complete, the observer used a cursor to indicate which of the three balls had been shown. In the second part, the observers estimated distance to the simulated object; they did so by moving a cursor to the desired position on a scale that was labeled $4 \mathrm{ft}$ at the top and $54 \mathrm{ft}$ at the bottom with labeled tick marks corresponding to 2 - $\mathrm{ft}$ intervals. No feedback was given following either the size or the distance judgments. Observers were given up to 12 practice trials (without feedback) before beginning each part of the experiment.

\section{Results and Discussion}

Figure 3 displays the observers' average percentage of correct identifications for each ball type. Chance performance would yield $33 \%$ correct, so the observers' identifications were better than chance for all three ball types. Table 1 presents the distributions of observer responses for each simulated ball type. Performance was clearly better than chance $\left[\chi^{2}(4)=184.99, p<.0001\right]$, but the observers made many errors. In particular, they were inclined to report that the child's basketball was presented more often and that the adult basketball was presented less often than actual frequencies. The $d^{\prime}$ values were 0.23 for the adult basketball, 0.55 for the child's basketball, and 0.99 for the softball (Hacker \& Ratcliff, 1979).

Distance estimates were reasonably accurate. Figure 4 displays the observers' average distance estimates as a function of the simulated distance. Estimated distance increases monotonically with simulated distance, but as in most distance estimation tasks, the estimated distance tends to saturate at longer simulated distances (Gilinsky, 1951; Rieser, Ashmead, Talor, \& Youngquist, 1990). The correlation between simulated and judged distance was .66. A repeated measures analysis of variance (ANOVA) with judged distance as the dependent variable revealed a significant effect of simulated distance $[F(4,28)=$ $116.76, p<.0001]$. We also analyzed the data from the whole trajectory and falling trajectory trials separately, but found no differences in the accuracy of size or distance judgments.

The results from this experiment show that observers were able to identify the absolute size and distance of simulated freely falling objects. We cannot conclude with certainty, however, that they were using the dynamics of the objects' motion to make these judgments, because the stimuli provided other cues associated with simulated distance. For example, because the object trajectories always subtended the same visual angle, longer stimulus durations were always associated with larger simulated distances.

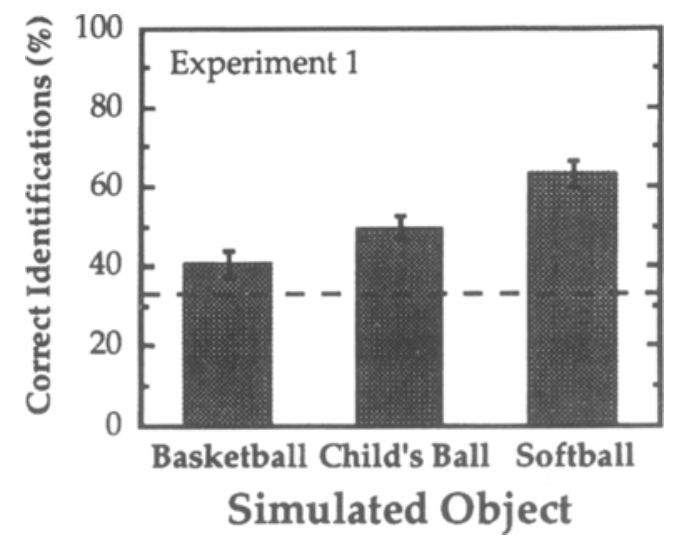

Figure 3. Identifications of ball type in Experiment 1. The percentage of correct identifications is plotted for each simulated ball type. The percentage associated with chance performance is $33.3 \%$ and is represented by the dashed line. The error bars indicate standard errors of the mean. 
Table 1

Distribution of Ball Type Responses in Experiment 1

\begin{tabular}{|c|c|c|c|c|c|c|}
\hline \multirow[b]{3}{*}{ Response } & \multicolumn{6}{|c|}{ Simulated Ball Type } \\
\hline & \multicolumn{2}{|c|}{ Basketball } & \multicolumn{2}{|c|}{ Child's Ball } & \multicolumn{2}{|c|}{ Softball } \\
\hline & $n$ & Prop. & $n$ & Prop. & $n$ & Prop. \\
\hline Basketball & 97 & .40 & 69 & .29 & 16 & .07 \\
\hline Child's ball & 117 & .49 & 119 & .49 & 72 & .30 \\
\hline Softball & 26 & .11 & 52 & .22 & 152 & .63 \\
\hline Total & 240 & & 240 & & 240 & \\
\hline
\end{tabular}

Note- $n$, number of responses; Prop., proportion.

In addition, projected size of the objects was correlated with simulated distance and simulated size $(r=-.71$ and .71 , respectively), so observers' judgments might have been based on projected size.

In Experiment 2, we examined the possibility that observers based their estimates on stimulus duration. This was accomplished by varying the height of the apex of the ball's flight, making duration an unreliable indicator of simulated distance. In Experiment 3, we examined the possibility that observers' judgments were based on projected size. This was accomplished by adding a static condition in which the objects were presented in fixed position.

\section{EXPERIMENT 2}

\section{Method}

Subjects. Eight male observers, none of whom had participated in Experiment 1, were paid for their participation. They had normal or corrected-to-normal vision and were again not informed of the experimental hypotheses. With the addition of variations in apex height, there were 45 different stimulus presentations ( 3 ball sizes $\times 5$ distances $\times 3$ apex heights); each was presented once.

Stimuli and Procedure. The methods and procedure of Experiment 2 were the same as those of Experiment 1, with the following exceptions. First, the apex height was varied from trial to trial, thereby decoupling stimulus duration from simulated distance; three apex heights were presented, corresponding to the top of the display screen and 1/4 and 1/2 of the screen height from the top. Second, because Experiment 1 revealed no difference in performance between rise-and-fall trajectories as compared with fall trajectories, we only presented rise and fall trajectories. Third, the observers made two judgments - first, size, and then, distance-after each stimulus presentation. (The observers were given a good deal of practice, so they were able to make combined judgments as accurately as separate judgments.) Fourth, a second block of trials was presented, in which the animation sequence consisted of a rise and fall of the simulated object at constant velocity (except for the reversal of direction at the apex). The same constant velocity was used for all simulated objects. We added this block in order to examine the contribution of stimulus motion cues other than acceleration to observers' size and distance judgments. The durations of the constant-velocity stimulus presentations were somewhat longer than those of the accelerating stimuli. The trajectories of the simulated objects in the accelerating and constant-velocity conditions are displayed in Figure 5. The image velocity of the ball was always perfectly predictive of the simulated distance in the constant-velocity condition.

\section{Results and Discussion}

Figure 6 shows the percentage of correct identifications of the three ball types across all trials in Experi- ment 2; the bars labeled "accelerating" are the values for trials in which the stimulus motion corresponded to a frictionless rise and fall, and the points labeled "constant velocity" are the values for trials in which the stimulus motion consisted of a constant velocity (with a reversal of direction at the apex). Observers' identifications were better than chance for all three ball types for both accelerating $\left[\chi^{2}(4)=75.48, p<.0001\right]$ and constant-velocity stimuli $\left[\chi^{2}(4)=63.06, p<.0001\right]$. The percentage of correct identifications was generally higher in the accelerating than in the constant velocity trials. We also calculated $d^{\prime}$ for each condition. With accelerating stimuli, $d$ 's were $0.34,0.70$, and 0.80 for the basketball, child's ball, and softball, respectively. With constant velocity stimuli, they were $0.23,0.41$, and 0.63 . A one-tailed $t$ test $[t(4)=$ $1.44, p=.0995]$, based on the mean of absolute size judgment errors, revealed a nonsignificant trend for errors associated with accelerating balls (mean error $=3.76 \mathrm{~cm}$ ) to be smaller than errors for constant velocity (mean error $=4.11 \mathrm{~cm}$ ). These findings suggest that observers make some use of the additional size information conveyed by the acceleration profiles as opposed to the average velocity profiles. However, the advantage provided by acceleration is small.

Figure 7 shows the relationship between simulated and estimated distance for the two types of stimuli presented in Experiment 2. As with the size judgments, the observers' distance estimates were reasonably accurate with the accelerating stimuli and slightly less accurate with the constant-velocity stimuli: the correlations between judged and simulated distance were .67 and .59 for the accelerating and constant-velocity stimuli, respectively. A comparison between absolute distance judgment errors showed that accelerating stimuli (mean error $=2.67 \mathrm{~m}$ ) were judged somewhat more accurately than constant-velocity stimuli (mean error $=3.15 \mathrm{~cm}$ ) $[t(4)=3.09, p=.011]$. A repeated measures ANOVA was conducted with sim-

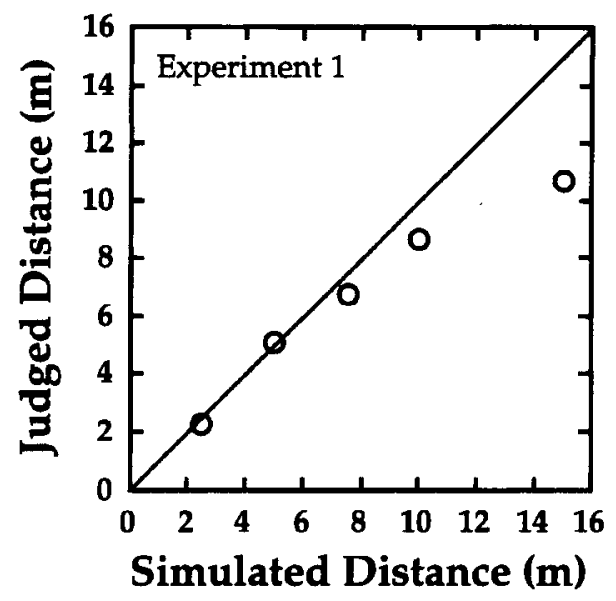

Figure 4. Distance judgments in Experiment 1. The estimated distance is plotted as a function of the simulated distance in meters. The diagonal line corresponds to veridical estimates. Error bars indicating standard errors are smaller than the symbols. 


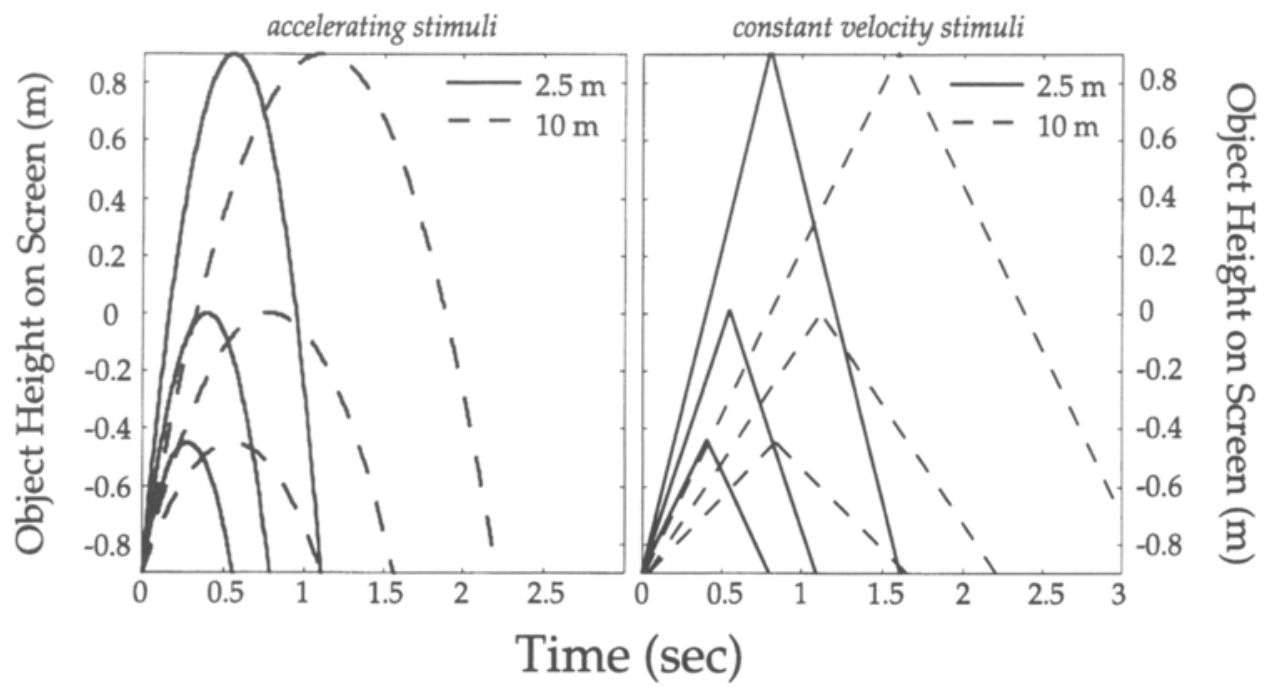

Figure 5. Projected trajectories in Experiment 2. Vertical position on the display screen is plotted as a function of time. The left panel shows the trajectories for the accelerating condition for simulated distances of 2.5 and $10 \mathrm{~m}$. There were three different apices in this experiment, one at the top of the screen, one of them three quarters of the way to the top, and one of them half of the way. The right panel shows the trajectories for the constant-velocity condition for simulated distances of 2.5 and $10 \mathrm{~m}$. Again there were three different apices.

ulated distance, apex height, and correctness on size judgment as factors, and judged distance as the dependent measure. There was a significant main effect of simulated distance $[F(4,28)=55.13, p<.0001]$. The main effect of apex height was not significant, implying that observers did not base their distance judgments on final velocity or stimulus duration. Thus, it appears that acceleration information provides a small advantage over velocity when an observer estimates object distance.

We were also interested in determining whether distance judgments were more or less accurate when object size had been correctly identified than when it had not.

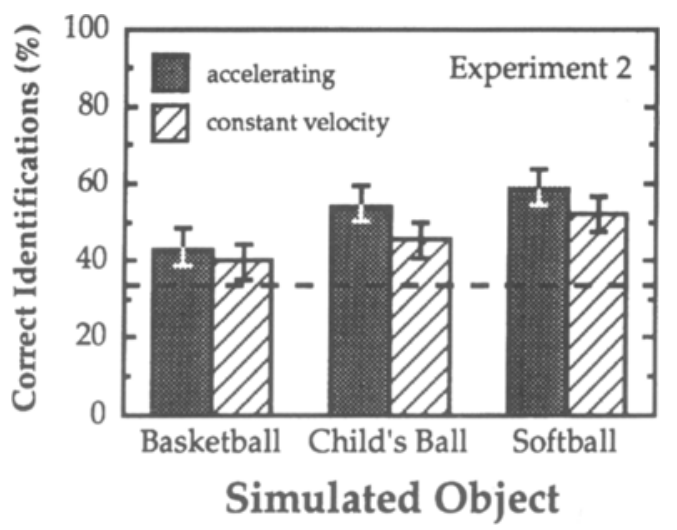

Figure 6. Identifications of ball type in Experiment 2. The percentage of correct identifications is plotted for each simulated ball type. Solid bars represent identification performance with accelerating stimuli, and striped bars represent performance with constant-velocity stimuli. The percentage associated with chance performance is $33.3 \%$ and is represented by the dashed line. The error bars indicate standard errors.
An ANOVA revealed that distance judgments were more accurate when size had been identified correctly $[F(1,7)=$ $3.83, p<.051]$. Moreover, the correlation coefficients between simulated and judged distance were .71 for accelerating stimuli whose size had been correctly identified and .63 for accelerating stimuli whose size had not been correctly identified. Thus, distance judgments tended to be more accurate when size was identified correctly.

\section{EXPERIMENT 3}

As previously mentioned, there was a correlation between projected size and simulated distance $(r=-.71)$ as well as between projected size and simulated object size $(r=.71)$ in Experiments 1 and 2. Thus, it is possible that observers' distance and size judgments were based on the projected size of the objects rather than on their motion properties. Experiment 3 was designed to test this possibility. This was accomplished by adding a static condition in which the simulated objects were presented in fixed position. In Experiment 3, we also examined whether the apex of the object's trajectory would be critical for use of the gravity cue. This was done by comparing observers' judgments in the nonstatic conditions, with the apex visible and with the apex invisible.

\section{Method}

Subjects. Eight male observers (ages 18-42) were paid for their participation. They had normal or corrected-to-normal vision and were not informed of the experimental hypotheses. None had participated in Experiment 1 or 2.

Stimuli. The stimuli were identical to those in Experiment 2, with the following exceptions. First, three movement conditionsaccelerating, constant velocity, and static - were presented to each observer. The accelerating and constant-velocity conditions were 


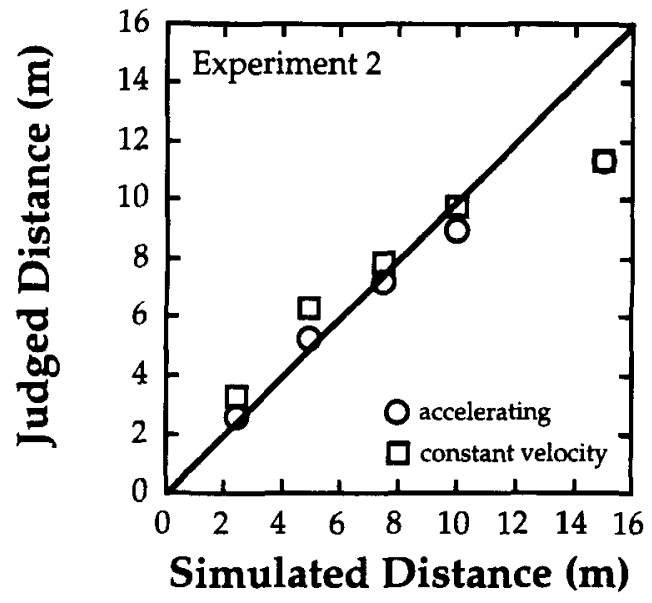

Figure 7. Distance judgments in Experiment 2. Estimated distance is plotted as a function of simulated distance in meters. Circles represent judgments with accelerating stimuli, and squares judgments with constant-velocity stimuli. The diagonal line corresponds to veridical estimates. Error bars indicating standard errors are smaller than the symbols.

the same as those in Experiment 2. In the static condition, the simulated balls were presented in fixed position at the center of the display screen for $2 \mathrm{sec}$. Second, in the accelerating and constant velocity conditions, the trajectories were presented an equal number of times with apex visible or not visible. When the apex was not visible, the ball would rise, leave the top of the screen, reenter the screen, and fall. Third, stimuli were presented in three blocks. The first was the accelerating condition, in which the ball's motion was the canonical deceleration and acceleration pattern used in Experiment 1 . Trials with apex visible and invisible were presented in random order. The first block consisted of 90 stimulus presentations.
The second block was the constant-velocity condition, in which the balls moved at the average velocity of the first block (with a reversal of direction at the top). Again, trials with the apex visible and with the apex invisible were presented in random order. This block consisted of 90 trials. The third block was the static condition, in which the stimuli were presented in fixed position at the center of the screen for $2 \mathrm{sec}$. This block consisted of 45 stimulus presentations. Blocks 1 and 2 were counterbalanced between subjects; Block 3 was always presented last. Fourth, the durations of the constantvelocity and accelerating stimulus presentations were equated. Figure 8 shows height over time for two example stimuli (2.5- and 10 -m distances) for all three conditions.

Procedure. The procedure was the same as in Experiment 2. The observers were given up to 12 practice trials (without feedback) before beginning the experiment. In the experiment, the observers made a size judgment and then a distance judgment. No feedback was given.

\section{Results and Discussion}

Figure 9 shows the percent of correct identifications for the three ball types across all trials in Experiment 3. The bars labeled "accelerating" depict the values for trials in which the stimulus motion corresponded to the canonical deceleration and acceleration pattern. Those labeled "constant velocity" show the values for constantvelocity trials. Finally, the bars labeled "static" represent the percentage of correct identifications for trials in which the simulated objects did not move. There was no difference in identification accuracy between apex-visible and apex-invisible trials, so those data have been averaged to produce the accelerating and constant-velocity values. Observers chose the correct ball in all three conditions more frequently than expected for chance performance [accelerating, $\chi^{2}(4)=252.8, p<.0001$; constant

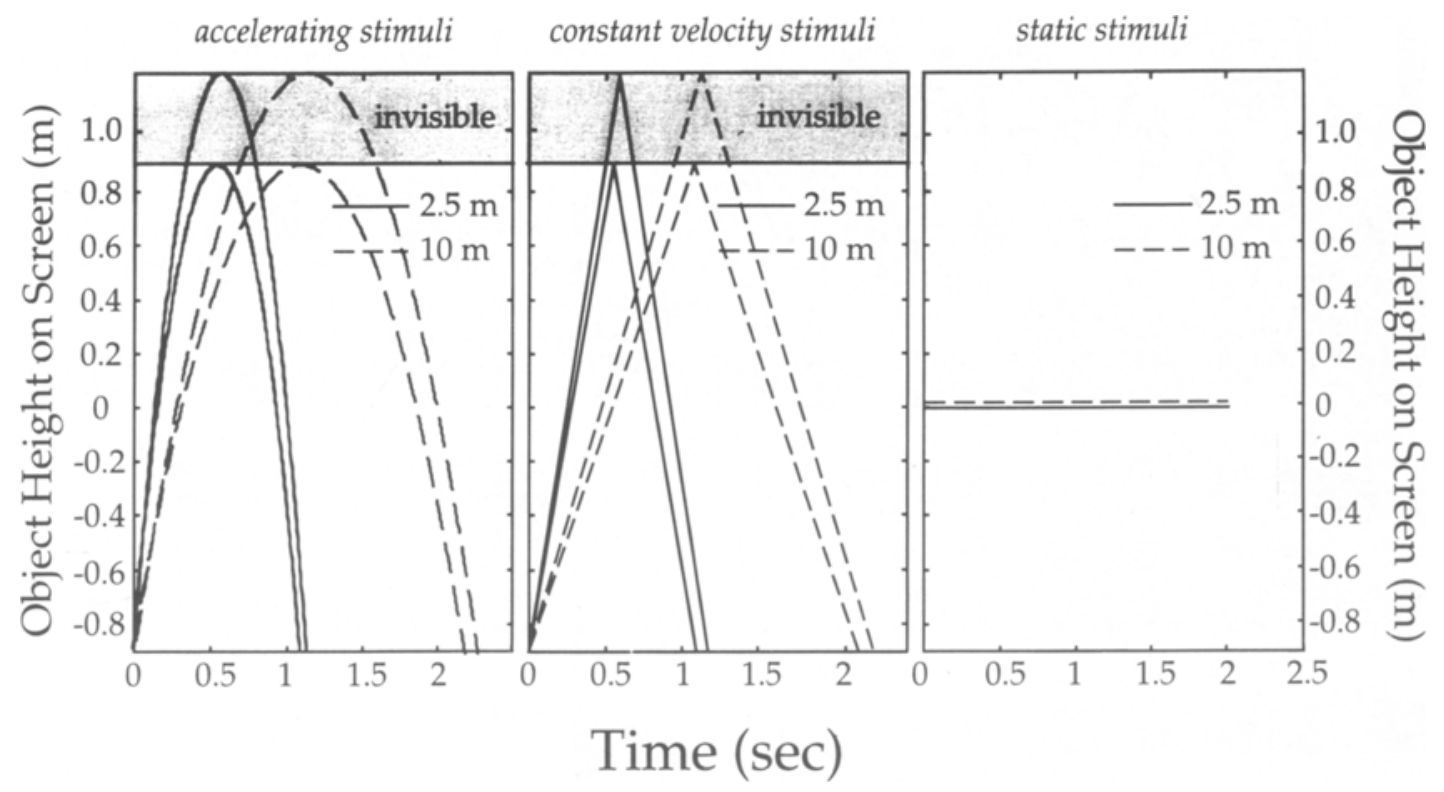

Figure 8. Projected trajectories in Experiment 3. Vertical position on the display screen is plotted as a function of time. The left panel shows the trajectories for the accelerating condition for simulated distances of 2.5 and $10 \mathrm{~m}$. There were two different apices in this experiment, one at the top of the screen and one above the top; the apex in the latter case was invisible. The center panel shows the trajectories for the constant-velocity condition for simulated distances of 2.5 and $10 \mathrm{~m}$. 


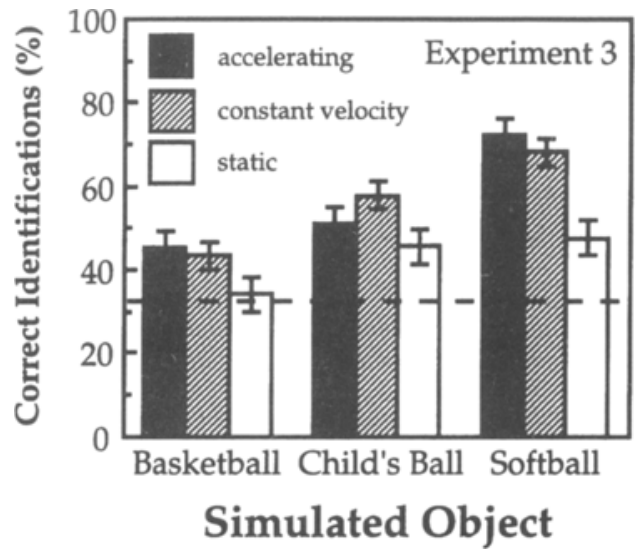

Figure 9. Identifications of ball type in Experiment 3. The percentage of correct identifications is plotted for each simulated ball type. Solid bars represent identification performance with accelerating stimuli; striped bars, performance with constantvelocity stimuli; and white bars, performance with static stimuli. The percentage associated with chance performance is $33.3 \%$ and is represented by the dashed line. The error bars indicate standard errors.

velocity, $\chi^{2}(4)=229.9, p<.0001$; static, $\chi^{2}(4)=25.1$, $p<.0001]$. There was no significant difference in performance between the accelerating and constant-velocity conditions. The percentage of correct identifications was above chance in the static condition, but was significantly lower than in the two moving conditions [accelerating vs. static, $\chi^{2}(4)=326.4, p<.0001$; constant vs. static, $\left.\chi^{2}(4)=262.5, p<.0001\right]$. The same pattern of results was revealed by the correlations among simulated, projected, and judged sizes that are shown in Table 2. Observers were able to use the correlation between projected and simulated size in making size judgments, but their judgments were more accurate when motion information was present. Unlike the finding of Experiment 2, size judgments were no better in acceleration than in constant velocity trials.

Figure 10 shows the relationship between simulated and estimated distance for the three conditions presented in Experiment 3. There were no differences in the accuracy of distance estimates in the moving conditions when the apex was visible or not, so those data have been averaged. As with the size judgments, the observers' distance estimates were reasonably accurate in the moving stimulus conditions: The correlations between judged and simulated distance were .62 and .70 for the accelerating and constant-velocity stimuli, respectively. Estimates were less accurate, but better than chance, in the static condition: the correlation between judged and simulated distance was .37 .

A repeated measures ANOVA was conducted with simulated distance and type of motion as independent variables and judged distance as the dependent variable. Unlike the finding of Experiment 2, performance was not different in the accelerating and constant-velocity conditions. Observers' judgments were significantly more accurate in the constant-velocity condition than in the static condition
$[F(1,7)=21.3, p=.0024]$ and in the accelerating condition than in the static condition $[F(1,7)=19.7, p=.003]$.

The results from the static condition show that observers could indeed use the correlations between projected and simulated size and between projected size and simulated distance in making size and distance judgments. However, the accuracy of size and distance judgments was systematically higher in the accelerating and constantvelocity conditions than in the static condition, so observers also utilized the information contained in the simulated objects' motion.

Watson et al. (1992) suggested that the apex is a particularly informative segment of the object's trajectory. The data are inconsistent with this hypothesis, in that judgments were no poorer when the apex was invisible. This is somewhat surprising, given that (1) the assumptions underlying the equations of Saxberg (1987a) and Watson et al. (1992) are most valid near the apex (where the drag force of air is negligible) and (2) proportional velocity changes are greatest near the apex and, therefore, more likely to be detected. Perhaps observers do not capitalize on the superior information of the apex because they simply do not make efficient use of acceleration information in general.

\section{EXPERIMENT 4}

If observers are in fact sensitive to gravitational acceleration, or if they are at least able to exploit other simpler motion cues that are correlated with gravitational acceleration, then displays of falling objects should lead to better performance than should displays of horizontally moving objects. We carried out a short study to test whether size/distance judgments based on vertical acceleration would be superior to those based on horizontal acceleration.

\section{Method}

Subjects. Eight male observers (ages 17-40) were paid for their participation. They had normal or corrected-to-normal vision and were not informed of the experimental hypotheses. None had participated in Experiments 1-3.

Stimuli. The stimuli were mostly identical to those in Experiment 3 . That is, constant velocity stimuli, accelerating, and static ones were used. The apex was always visible. All stimuli were duplicated moving from left to right with identical motion parameters.

Procedure. The procedure of Experiment 3 was used, with the following exceptions. The size judgments were dropped. Horizontal motions were added. Four observers saw the complete set of vertical trials first and the other 4 saw the horizontal trials first. The static trials were always presented last. Within these constraints, the orders of constant velocity and acceleration were fully counterbalanced.

Table 2

Correlation Table for Judged Size, Experiment 3

\begin{tabular}{lccc}
\hline & \multicolumn{3}{c}{ Simulated Motion Type } \\
\cline { 2 - 4 } \multicolumn{1}{c}{ Correlations } & Acceleration & Constant Velocity & Static \\
\hline Judged-simulated size & .56 & .51 & .20 \\
Judged-projected size & .41 & .30 & .43 \\
Projected-simulated size & .44 & .44 & .44 \\
\hline
\end{tabular}




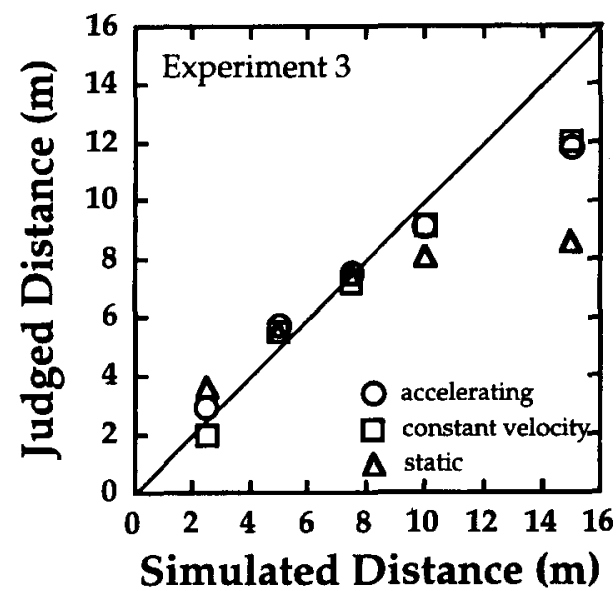

Figure 10. Distance judgments in Experiment 3. Estimated distance is plotted as a function of simulated distance in meters. Circles represent judgments with accelerating stimuli; squares, judgments with constant-velocity stimuli; and triangles, judgments with static stimuli. The diagonal line corresponds to veridical estimates. Error bars indicating standard errors are smaller than the symbols.

\section{Results and Discussion}

We observed small differences in the ability to judge size and distance with vertical and horizontal motions. Because of large individual differences, the following analyses were performed. First, individual correlation coefficients between judged and simulated distance were computed for each condition. The averages across subjects were as follows: $r=.61$ (vertical, accelerating), $r=.57$ (horizontal, accelerating), $r=.75$ (vertical, constant velocity), $r=.67$ (horizontal, constant velocity), and $r=.43$ (static stimuli). Correlations were higher for constant velocity trials when the motion was vertical as opposed to horizontal $[t(7)=2.288, p=.056]$. The correlations for accelerating trials were not significantly different for the vertical and horizontal accelerations $[t(7)=1.28, p=.239]$.

Performance was fairly similar for vertical and horizontal motion, which suggests that vertical motion does not play a special role in the judging of object distance or size.

\section{EXPERIMENT 5}

Only three balls of rather different sizes were used in Experiments $1-4$, so there was a clear correlation between projected size and simulated size $(r=.71)$ and between projected size and simulated distance $(r=-.71)$. We were concerned that the presence of this correlation might have reduced the observers' willingness to use motionbased cues to judge size and distance. To reduce these correlations, the range of simulated sizes was tripled; nine different ball sizes were presented at six distances. The negative correlation between simulated distance and pro- jected size was thereby reduced to $r=-.45$. In addition, the closest simulated distance was doubled (to $5 \mathrm{~m}$ ), because the old value was close to the actual distance to the projection screen $(3 \mathrm{~m})$ and might have served as an anchor for distance judgments. We hoped that these changes would increase the observers' willingness to use motionbased cues to size and distance.

\section{Method}

Subjects. Eight volunteer observers ( 2 female and 6 male) who had not participated in Experiments $1-4$ were used. They comprised 2 of the authors (M.S.B. and H.H.) and 6 graduate students.

Stimuli. The same rear projection system was used as before. For the simulations, nine different ball sizes were presented at six distances. Table 3 shows the projected sizes for all stimuli as a function of simulated distance and size. With the increased stimulus range, the correlation between simulated distance and projected size was reduced to $r=-.45$. The size judgment task also became more difficult, because it was no longer possible to associate each simulated ball with a typical real-world ball. Constant-velocity trials and accelerating trials were equated in their average velocity. As before, the block of static stimuli was presented last.

Procedure. The procedure of Experiment 3 was used, with the following exceptions. The subjects were not informed about the range of simulated sizes or distances, but they were told that balls of many different sizes and at many different distances would be presented. Distance judgments were collected on an analogue rating scale, ranging from 1 to 35 yards. Size judgments were dropped, as in Experiment 4.

\section{Results and Discussion}

No significant differences in judgment errors were found between accelerating and constant-velocity stimuli. Correlations between simulated and judged distance were actually higher for constant-velocity $(r=.91)$ than for accelerating stimuli $(r=.81)$. Both were far superior to judgments of static stimuli $(r=.50)$, on the basis of a repeated measures ANOVA of absolute judgment errors $[F(2,14)=22.0, p<.0001]$. The individual correlations between simulated and judged distance are listed in Table 4 for each motion condition.

The slight superiority of acceleration trials found in Experiment 2 was not replicated. It appears that observers do not exploit the size/distance information carried by acceleration per se. Rather, they seem to utilize the information carried by average velocity during a stimulus presentation.

Table 3

Projected Sizes (in Meters), Experiment 5

\begin{tabular}{cccccccccc}
\hline & \multicolumn{10}{c}{ Simulated } & \multicolumn{10}{c}{ Size } \\
\cline { 2 - 10 } Distance & .05 & .07 & .10 & .14 & .20 & .28 & .40 & .56 & .80 \\
\hline 5 & .06 & .08 & .12 & .16 & .23 & .32 & .46 & .65 & .92 \\
7 & .04 & .06 & .08 & .12 & .16 & .23 & .32 & .46 & .65 \\
10 & .03 & .04 & .06 & .08 & .12 & .16 & .23 & .32 & .46 \\
14 & .02 & .03 & .04 & .06 & .08 & .12 & .16 & .23 & .32 \\
20 & .014 & .02 & .03 & .04 & .06 & .08 & .12 & .16 & .23 \\
28 & .01 & .014 & .02 & .03 & .04 & .06 & .08 & .12 & .16 \\
\hline
\end{tabular}

Note-Simulated distance is in meters. 
Table 4

Correlation Table for Judged and Simulated Distance, Experiment 5

\begin{tabular}{cccc} 
& \multicolumn{3}{c}{ Simulated Motion Type } \\
\cline { 2 - 4 } Subject & Acceleration & Constant Velocity & Static \\
\hline J. & .825 & .871 & .464 \\
K. & .841 & .89 & .494 \\
M. & .863 & .912 & .496 \\
R. & .755 & .956 & .498 \\
S. & .742 & .919 & .447 \\
B. & .746 & .962 & .551 \\
H. & .867 & .935 & .524 \\
W. & .871 & .912 & .569 \\
\hline
\end{tabular}

\section{GENERAL DISCUSSION}

\section{Comparison to Previous Results}

As mentioned earlier, Saxberg (1987b) tested observers' use of the gravity cue experimentally. He presented computer displays of balls propelled from below a textured ground plane with different initial velocities. Observers were asked to move a mouse-driven plate on the computer screen to "catch" the ball when it landed. Saxberg reported that these observers were reasonably accurate when the ball's position and projected size changed appropriately over time. However, when the projected size of the ball was held constant, performance suffered significantly. From this, he concluded that human observers are not sensitive to the distance information contained in the dynamics of free fall.

Watson et al. (1992) criticized Saxberg's (1987b) conclusion by arguing that the fixed size condition put two potentially useful cues - position over time and projected size over time-into conflict. On most trials, the trajectory information specified that a depth change occurred while the constant projected size specified that none occurred. Saxberg's observers, therefore, had to reconcile two conflicting sources of depth information in making their responses. For this reason, Watson et al. argued that Saxberg's results do not necessarily imply that human observers do not make use of the distance information conveyed by the dynamics of free fall. The results of the static condition in our Experiments 3 and 4 support the argument of Watson and colleagues indirectly. Observers' size and distance judgments were better than chance in that condition, so they clearly made use of the distance information contained in the projected sizes of the simulated objects. Size and distance judgments were more accurate, however, in the accelerating and constant-velocity conditions, which shows that observers made additional use of the distance information contained in the objects' motion. Thus, it appears that human observers use both projected size and motion cues in making size and distance estimates.

\section{Did Observers Use the Acceleration Information?}

We were unable to isolate the acceleration cue completely in our experimental situation, so other distance and size cues were available. These include projected size (correlated negatively with simulated distance and positively with simulated size), average image velocity (correlated negatively with simulated distance), and stimulus duration (correlated positively with simulated distance). Experiments 2-5 were designed in order to determine whether these cues aided distance and size estimation in our task.

In Experiment 2, we varied the height of the object's trajectory to render the duration cue less reliable and found that size and distance judgments were unaffected. This result shows that observers did not simply rely on stimulus duration.

In Experiments 2-5, we presented a constant-velocity condition and found that observers' size and distance judgments in that condition were significantly better than chance and essentially the same as in the acceleration condition. This result shows that observers can, in fact, make use of the average velocity cue. Although the results of Experiment 2 suggest that observers might make additional use of the information conveyed by object acceleration, this result could not be replicated in Experiments 3,4 , and 5 .

In Experiments 3, 4, and 5, we presented a static condition in which projected size was the only cue to simulated size and distance. Observers' judgments were better than chance, which shows that they can use projected object size to estimate size and distance. However, observers clearly made additional use of the information conveyed by object motion, as is demonstrated by the finding that their judgments were significantly more accurate in the accelerating and constant-velocity conditions than in the static condition.

Thus, the pattern of results across experiments is consistent with the hypothesis that human observers utilize the absolute distance and size information conveyed by image motion of a rising and falling object. However, there is no consistent evidence for use of information specific to acceleration and deceleration as opposed to average velocity during a stimulus presentation.

\section{Why Isn't Performance Better in These Tasks?}

Our results do not really support the gravity-cue hypothesis, because performance fell far short of what one would expect from efficient use of the cue. Specifically, a perfect correlation exists between acceleration and simulated distance, such that, in principle, the cue could provide highly accurate distance estimates. In fact, the distance estimates in the accelerating conditions of Experiments $1-5$ were only moderately accurate. Here, we will consider three reasons for the inability to exploit information provided by acceleration due to gravity.

First, size and distance judgments may have been adversely affected by the inability to sense object acceleration accurately, as is suggested by several psychophysical studies. For example, the proportion of velocity change required to distinguish accelerating from nonaccelerating motions ranges from 20\% (Babler \& Dannemiller, 1993) 
to nearly $100 \%$ (Calderone \& Kaiser, 1989; Rosenbaum, 1975). This lack of sensitivity to velocity change would lead to a similar estimation error of the object's distance.

Second, our experimental procedure, which involved passive viewing, did not require a distance-appropriate response from the observers. Perhaps observers would be better able to demonstrate their knowledge of absolute size and distance in active tasks. For example, a catching task might reveal better use of the information in our displays.

Third, the gravity cue may only be used qualitatively, because the dynamics of free fall are much more complicated in the real world than is implied by the equations of Saxberg (1987a) and Watson et al. (1992), which assume that object trajectories are unaffected by the drag force of air. Drag increases geometrically with object velocity. For a baseball moving at $80 \mathrm{miles} / \mathrm{h}$, the drag is about $70 \%$ of the ball's weight (Brancazio, 1985). Thus, Saxberg's and Watson's equations are only approximations for slower moving objects.

Thus, the visual system, instead of having to adjust for drag, might have adopted a cruder mechanism, reflecting the fact that an object moving at constant velocity gives rise to higher retinal image velocities at closer distances. This relationship usually holds, no matter how the object moves or whether the object is accelerating or moving at constant velocity. Presumably, observers are sensitive to this fundamental relationship, as was shown by their size/ distance judgments in the constant-velocity condition.

\section{SUMMARY AND CONCLUSION}

We have shown that image motion is used as a cue to absolute size and distance. In particular, when acceleration or constant velocity information is provided, judgments of absolute size and distance are much better than judgments based on static information alone. However, observers seem unable to use the size and distance information provided by object acceleration as opposed to velocity. Thus, our data are mostly inconsistent with the gravity-cue hypothesis as stated by Saxberg (1987a) and Watson et al. (1992).

\section{REFERENCES}

Babler, T. G., \& DANNEmiller, J. L. (1993). Role of image acceleration in judging landing location of free-falling projectiles. Journal of Experimental Psychology: Human Perception \& Performance, 19, 15-31.
Brancazio, P. J. (1985). Looking into Chapman's homer: The physics of judging a fly ball. American Journal of Physics, 53, 849-855.

Calderone, J. B., \& Kaiser, M. K. (1989). Visual acceleration detection: Effect of sign and motion orientation. Perception \& Psychophysics, 45, 391-394.

Cumming, B. G., Johnston, E. B., \& Parker, A. J. (1991). Vertical disparities and perception of 3-dimensional shape. Nature, 349 , 411-413.

Fisher, S. K., \& CiUfFreda, K. J. (1988). Accommodation and apparent distance. Perception, 17, 609-621.

Gilinsky, R. (1951). Perceived size and distance in visual space. Psychological Review, 58, 460-482.

Gillam, B., \& LAWERGRen, B. (1983). The induced effect, vertical disparity, and stereoscopic theory. Perception \& Psychophysics, 34, 121-130.

HACKER, M. J., \& RATClifF, R. (1979). A revised table of $d^{\prime}$ for Malternative forced choice. Perception \& Psychophysics, 26, 168-170.

Johansson, G., \& JansSON, G. (1967). The perception of free fall. Unpublished seminar paper, University of Uppsala, Sweden, Department of Psychology.

Kellman, P. J., \& Kaiser, M. K. (1995). Computing target motion without distance information: Combining constraints from optic flow and binocular disparity. Journal of the Optical Society of America A, 12, 623-625.

Longuet-Higgins, H. C., \& MaYhew, J. E. W. (1982). A computational model of binocular depth perception. Nature, 297, 376-378.

Muchisky, M. M., \& Bingham, G. P. (1992). Perceiving size in events via kinematic form. In J. K. Kruschke (Ed.), Proceedings of the 14th Annual Conference of the Cognitive Science Society (pp. 1002-1007). Hillsdale, NJ: Erlbaum.

Rieser, J. J., Ashmead, D. H., Talor, C. R., \& Youngquist, G. A. (1990). Visual perception and the guidance of locomotion without vision to previously seen targets. Perception, 19, 675-689.

Rogers, B. J., \& BradShaW, M. F. (1993). Vertical disparities, differential perspective and binocular stereopsis. Nature, 361, 253-255.

Rosenbaum, D. A. (1975). Perception and extrapolation of velocity and acceleration. Journal of Experimental Psychology: Human Perception \& Performance, 1, 395-403.

SAXBERG, B. V. H. (1987a). Projected free fall trajectories: I. Theory and simulation. Biological Cybernetics, 56, 159-175.

SAXBERG, B. V. H. (1987b). Projected free fall trajectories: II. Human experiments. Biological Cybernetics, 56, 177-184.

SpotTiswoode, R. (1969). The Focal encyclopedia of film and television techniques. New York: Hastings House.

TREsiLian, J. R. (1994). A note on "Gravity as a monocular cue for perception of absolute distance and/or absolute size." Perception, 23, $727-731$

von Hofsten, C. (1990). Catching. In H. Heuer \& A. F. Sanders (Eds.), Tutorials on perception and action (pp. 33-46). Hillsdale, NJ: Erlbaum,

Watson, J. S., Banks, M. S., von Hofsten, C., \& Royden, C. S. (1992). Gravity as a monocular cue for perception of absolute distance and/or absolute size. Perception, 21, 69-76.

(Manuscript received July 22, 1994; revision accepted for publication January $11,1996$. 